\title{
Occupational Exposure to Extremely Low Frequency Magnetic Field and Risk of Alzheimer Disease: A systematic Review and Meta-analysis
}

\author{
Hamed Jalilian ${ }^{1}$, Saeed Hosseini Teshnizi ${ }^{2}$, Martin Röösli ${ }^{* 3,4}$, Masoud Neghab ${ }^{4}$ \\ 1. Student Research Committee, Shiraz University of Medical Sciences, Shiraz, Iran \\ 2. Biostatistician, Social Determinants in Health Promotion Research Center, \\ Hormozgan University of Medical Sciences, Bandar Abbas, Iran \\ 3. Swiss Tropical and Public Health Institute, Socinstrasse 57, P.O. Box, CH-4002 \\ Basel, Switzerland \\ 4. University of Basel, Basel, Switzerland \\ 5. Research Center for Health Sciences, Institute of Health, Department of \\ Occupational Health Engineering, School of Health, Shiraz University of Medical \\ Sciences, Shiraz, Iran
}

*Corresponding author at: Department of Epidemiology and Public Health, Swiss Tropical and Public Health Institute, Socinstrasse 57, P.O. Box, CH-4002 Basel, Switzerland.

E-Mail: martin.roosli@unibas.ch 


\section{Abstract:}

Introduction: Occupational exposure to extremely low frequency magnetic fields (ELF-MF) occurs in many occupations such as welders, electric utility workers, train drivers and sewing machine operators. There is some evidence suggesting ELF-MF exposure to be a risk factor for Alzheimer's disease (AD). The current study aims at systematically reviewing the literature and conducting a meta-analysis to evaluate the risk of AD amongst workers exposed to ELF-MF.

Methods: Bibliographic databases were searched including PubMed, EMBASE, Cochrane Library, and Web of Science in November 2017. Risk of bias was assessed in the all included studies. Pooled estimates were obtained using random-effects meta-analysis. In addition, sources of heterogeneity between studies and publication bias were explored.

Results: In total, 20 articles met the inclusion criteria. The pooled results suggest an increased risk of $\mathrm{AD}$ (RR: 1.63; 95\% CI: 1.35, 1.96). Higher risk estimates were obtained from casecontrol studies (OR: 1.80; 95\% CI: 1.40, 2.32) than from cohort studies (RR: 1.42; 95\% CI: 1.08, 1.87). A moderate to high heterogeneity $\left(\mathrm{I}^{2}=61.0 \%\right)$ and indication for publication bias (Egger test: $\mathrm{p}<0.001)$ were found.

Conclusion: The results suggested that occupational exposure to ELF-MF might increase the risk of $\mathrm{AD}$. However, this suggestion should be interpreted with caution given the moderate to high heterogeneity and indication for publication bias.

Key words: Alzheimer disease, Magnetic fields, Occupational exposure, Systematic review, Metaanalysis 


\section{Introduction}

Due to industrialization, characterized by an increasing production, distribution, and consumption of electricity, occupational exposure to extremely low frequency magnetic fields (ELF-MF) in the frequency range of $3 \mathrm{~Hz}$ to $300 \mathrm{~Hz}$ (ACGIH, 2015) is common in many work environments. The exposure originates from overhead and underground power lines (Monazzam, M. et al., 2015; Monazzam, M.R. et al., 2015), different medical equipment (McRobbie, 2012), electrical appliances, and electrical motors (Eskelinen et al., 2016) for instances in trains (Jalilian et al., 2017; Monazzam et al., 2016). As a consequence, electrical power installers and repairers, electricians, electrician apprentices, power plant operators (Bowman et al., 2007; Jalilian et al., 2016; Monazzam, M.R. et al., 2015), welders, train drivers, spinners, weavers, knitters, dyers, tailors, and dressmakers (Seidler, Andreas et al., 2007) are occupationally exposed to these fields. Some organizations such as International Commission on Non-Ionizing Radiation Protection (ICNIRP) and American Conference of Governmental Industrial Hygienists (ACGIH) have established occupational exposure threshold limits for ELF-MF (whole body ceiling exposure limit in $60 \mathrm{~Hz}$ for ACGIH and ICNIRP: $1 \mathrm{mT}$ ) (ACGIH, 2015; ICNIRP, 2010).

ELF-MF is classified as a "possibly carcinogenic to humans" agent by the International Agency for Research on Cancer (World Health Organization, 2016) according to 'fairly consistent' findings from childhood leukemia studies (Ahlbom et al., 2001; Greenland et al., 2000). In adults, study results are less consistent although previous meta-analyses also reported overall increased risk for leukemia and brain cancer (Kheifets et al., 2008; Zhang et al., 2016), as well as various neurodegenerative rare diseases, in particular Alzheimer's disease (AD) (García et al., 2008). 
The first epidemiological study on AD was published by Sobel et al. (1995) who reported that occupational exposure to ELF-MF above $0.2 \mu \mathrm{T}$ might elevate the risk for AD (Sobel et al., 1995). Following this study, more attention was drawn to this disease and further epidemiological studies were conducted. Qiu et al. reported that the relative risk (RR) of AD in workers exposed to more than $0.2 \mu \mathrm{T}$ ELF-MF was higher $(2.30 ; 95 \%$ confidence intervals [CI]: $1.00,5.10$ ) than those exposed to less than $0.2 \mu \mathrm{T}$ (Qiu et al., 2004). Roosli et al. found a higher $\mathrm{AD}$ risk for train drivers and shunting yard engineers, which were highly exposed to $16.7 \mathrm{~Hz}$ compared to lower exposed station masters (Roosli et al., 2007).

However, some studies showed no significant relationship between exposure to ELF-MF and evaluated risk of AD. In 1999, Graves et al. reported an odds ratio (OR) of 0.95 (95\% CI: 0.27, 2.43) for $\mathrm{AD}$ for workers exposed to more than $0.3 \mu \mathrm{T}$ (Graves, Amy Borenstein et al., 1999). Sorahan and Mohammed showed no convincing evidence that United Kingdom (UK) electrical workers had suffered from elevated risk of AD due to ELF-MF exposure (Sorahan and Mohammed, 2014).

Some meta-analyses have addressed the overall association between occupational ELF-MF exposure and AD (García et al., 2008; Vergara et al., 2013). In a meta-analysis, Huss and Vermeulen reported a pooled RR of $1.51(95 \%$ CI: $1.21,1.88)$ for occupations with high vs. low average MF exposure. Strikingly, they found a consistent exposure-response pattern with studies including highly exposed occupations which reported the highest risk estimates (Huss and Vermeulen, 2014). Another meta-analysis, conducted by Vergara et al., indicated a small association (RR: $1.27 ; 95 \%$ CI: 1.15, 1.40) between occupational exposure to ELF-MF and AD risk (Vergara et al., 2013). Moreover, in 2008, Garcia et al. found pooled estimates of 2.03 (95\% 
CI: $1.38,3.00)$ and $1.62(95 \%$ CI: $1.16,2.27)$ for case-control and cohort studies, respectively (García et al., 2008).

Totally, Veagara et al. and Garcia et al. reported substantial heterogeneity between studies, for instances, between case-control and cohort studies. They also indicated gender differences in the risk estimates potentially reflecting different occupations for men and women. Quality of exposure assessment or outcome classification was also discussed to be a relevant source of bias producing heterogeneity among the studies. However, other relevant variables such as job title (the most exposed jobs) or frequency has not been systematically evaluated yet. In addition, to the best of our knowledge, four new or updated studies have been published and no metaanalysis has been conducted since 2013 (Vergara et al., 2013) (searching period is up to January 12, 2012). Therefore, the aim of this paper was to provide an updated systematic review including meta-analysis of occupational ELF-MF exposure and AD and to systematically address the source of heterogeneity reported in previous meta-analyses.

\section{Material and Methods}

\subsection{Search strategy}

A systematic literature search was conducted in the Cochrane Library, EMBAS, PubMed, and Web of Science databases up to November 1, 2017, using the following keywords: “neurodegenerative”, “Alzheimer”, “dementia”, in combination with “extremely low frequency”, "ELF", "EMF", "magnetic field", "electric field”, “electromagnetic", "occupation”, “job”, “workplace", "electrical occupation”, “electricity work", "power line”, "occupational exposure", “work-related", as well as "welder”, "railway”, “electric utility”, "power plant”, "welding”, and "garment". In addition, Medical Subject Headings (MeSH) include "neurodegenerative diseases", "Alzheimer's disease", “dementia" in combination with "magnetic fields", 
"electromagnetic fields", "occupations", "workplace", "work", and "occupational exposure" were searched in the PubMed and Cochrane Library databases. Moreover, the specialist literature database EMF-PORTAL (https://www.emf-portal.org) was checked using "Alzheimer" as the search term (2017). Additional studies were identified by manual search in Google, and from the references of original studies or review articles on the current topic.

\subsection{Study selection}

Two authors (H.J and S.H) evaluated all documents regarding the title, keywords and abstract. If they were not able to identify the articles by title and abstract, full-text was evaluated. When two reviewers were unable to reach an agreement, a third party (M.N) was included in the decision making process.

\subsection{Eligibility criteria}

Peer-reviewed articles that reported AD risk estimates in association with ELF-MF exposure were eligible for analysis. These studies must have included well-defined occupations or tasks related to ELF-MF exposure. ELF-MF exposure of the participants had to be assessed by measurements, estimated by job exposure matrix (JEM), or an industrial hygienist (IH). The outcome had to be defined as a medical diagnosis of $\mathrm{AD}$ or registered as $\mathrm{AD}$ on a death certificate. In the case of overlapping publications from the same study, the most comprehensive paper was used studies with poorly defined occupational groups (e.g. manual workers), or occupation unrelated to ELF-MF (e.g. fumes) were excluded. Furthermore, review articles, mechanism, laboratory or clinical studies, non-English articles, and non-occupational studies were excluded as well.

\subsection{Data extraction}


We extracted following three types of data from each study:

1. General data of studies: years of publication, Country, time period, number of cases, covariates, and study design.

2. The outcome data: the source of outcome data, method of assessment, and diagnosis creation.

3. The exposure/job data: the source of exposure/job data, exposure assessment method, and exposure creation.

We extracted quantitative risk estimates including ORs, RRs, and Hazard Ratios (HR) and their 95\% CIs of the AD risk for workers occupationally exposed to ELF-MF. The risk estimates of the highest ELF-MF exposure groups were extracted, if available. In addition, AD risk of electric occupations, welders, and train drivers were also extracted if available. For later subgroup comparisons, risk estimates of the following strata were extracted if available: gender (male,

female, and both genders combined), country (USA, Scandinavia, and other), exposure level $(>0.2 \mu \mathrm{T},>0.3 \mu \mathrm{T}$, and $>0.5 \mu \mathrm{T})$, frequency of electricity $(50$ and $60 \mathrm{~Hz}$ ), source of outcome data (death certificate, medical records, and other), and type of study (case-control and cohort studies).

\subsection{Risk of bias in individual studies}

We used a modified version of Repacholi et al. method (Repacholi et al., 2012) for assessment of bias in individual studies. Totally, in this section we pointed out six source of bias including funder, reporting, data analysis, selection-participation bias, confounding, and recall and information bias (supplemental table S1). They were weighted by two stars (full weight), one star (partial weight), and no star (no-weight), if applicable. Then, total risk of bias in individual 
studies was indicated by summing of stars, so that the highest and the lowest risk of bias were showed by zero star and twelve stars, respectively.

\subsection{Data analysis}

Overall, the $\mathrm{OR}$ of $\mathrm{AD}$ in case-control studies approximates RR. Therefore, when an association between ELF-MF and the risk of AD was estimated in the analyses of pooled studies, the OR was used as a RR measure. In addition, HR was considered as equal to RR. 


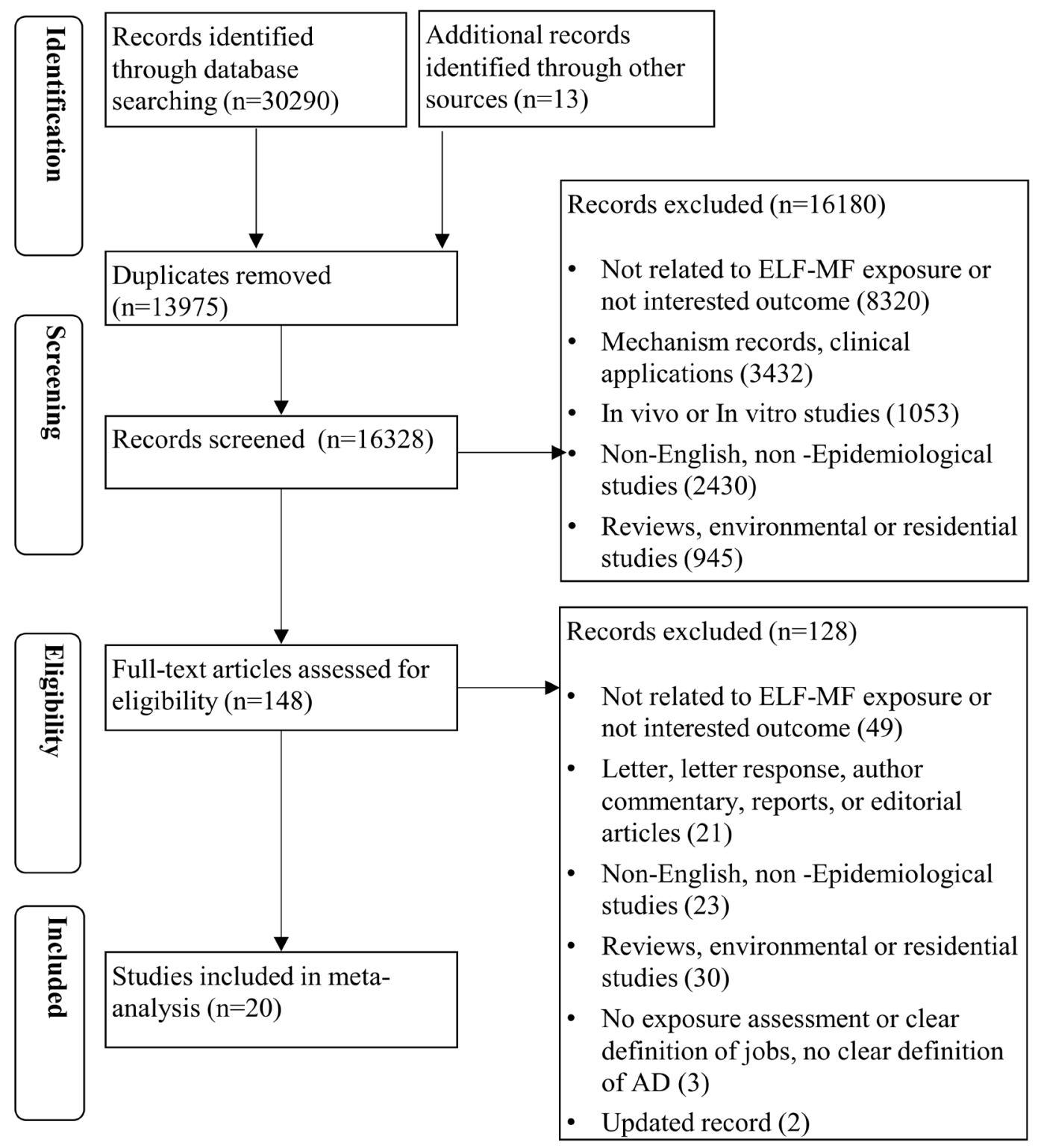

Figure 1. PRISMA flow diagram of article selection and exclusion for final analysis

After extracting quantitative information from each study, the effect size of each study was calculated based on the RR and standard error (SE) of RR. To evaluate the heterogeneity, Q-test $\left(\mathrm{p}<0.1\right.$ as heterogeneity) and the $\mathrm{I}^{2}$ statistics $(25 \%, 50 \%$, and $75 \%$, indicating low, moderate, and high levels of heterogeneity, respectively) were used. Also, sensitivity analysis was carried out to assess the robustness of our findings. Given the large heterogeneity between studies, final pooled 
risk estimates were calculated using random effects (Der Simonian-Laird method) metaanalysis. The forest plot for all studies and the pooled estimate with $95 \%$ CI were drawn. The influence of individual risk estimates (sensitivity analysis) was assessed by performing repeated meta-analyses with one study left out each time (supplementary Figure S1). In addition, we

assessed sensitivity of the overall pooled estimate when leaving out sets of studies based on bias score (Low: pooled estimate of studies with score of $>10$; Medium to high: pooled estimate of studies with score of $\leq 10$; High: pooled estimate of studies with score of $<10$ )

Subgroup analysis was conducted to compare the effect size (RR) in gender, exposure, country, source of outcome data, and study design. In addition, we used meta-regression analyses to investigate potential sources of heterogeneity. Moreover, a cumulative meta-analysis was applied as more recent study may be of higher quality. Funnel plot as a visual method and the Begg's test as a statistical test were used to evaluate the publication bias. Moreover, the trim-and-fill method was applied to provide a summary effect adjusted for publication bias. All analyses were carried out in STATA (version 14.0; Stata Corp, College Station, TX, USA).

\section{Results}

\subsection{Selected studies}

A total of 148 relevant records were identified through databases and other sources. Of all records, 19 review articles, 17 non-English articles, 30 articles with no relation to ELF-MF exposure, 19 articles with no relation to $\mathrm{AD}, 21$ letter, letter response, author commentary, report, or editorial articles, twelve environmental or residential studies, and six nonepidemiological studies were excluded. In addition, two articles that were updated in the later publications, two articles with no exposure assessment or clear definition of jobs, and a study with no clear definition of AD were excluded. Finally, 20 articles (Andel et al., 2010; 
Davanipour et al., 2014; Davanipour et al., 2007; Feychting et al., 2003; Feychting et al., 1998; Graves, A. B. et al., 1999; Håkansson et al., 2003; Harmanci et al., 2003; Koeman et al., 2015; Noonan et al., 2002; Park et al., 2005; Pedersen et al., 2017; Qiu et al., 2004; Roosli et al., 2007; Savitz et al., 1998a; Savitz et al., 1998b; Seidler, A. et al., 2007; Sobel et al., 1995; Sobel et al., 1996; Sorahan and Mohammed, 2014) (one of them was included three studies (Sobel et al., 1995)) were selected for the final analyses (Figure 1).

\subsection{Study characteristics}

Table 1 shows the study characteristics included in the review. The articles were published between 1995 and 2015, but periods for the case ascertainment of the case-control studies were between 1977 (Sobel et al., 1995) and 1998 (Davanipour et al., 2007; Park et al., 2005) although in three case-control studies (Harmanci et al., 2003; Seidler, A. et al., 2007; Sobel et al., 1996) no time periods had been reported. For cohort studies, follow-up time periods covered 1950 (a study from the USA) (Savitz et al., 1998b) until 2010 in the UK (Sorahan and Mohammed, 2014). In general, the studies were carried out in the USA (nine studies), Sweden (five studies), Finland (two studies), Switzerland (one study), Denmark (one study), Turkey (one study), German (one study), UK (one study), and the Netherlands (one study).

The number of included AD cases ranged from 25 (Roosli et al., 2007) to 2,000 in a Swedish study (Feychting et al., 2003). Of the 22 studies (extracted from 20 articles), 14 were casecontrol and eight cohort studies. The source of AD diagnosis included medical records (eight case-control studies), a register-based database (one cases-control study), death certificate (three cases-control and six cohort studies), hospital records (one cohort study), and population-based studies (two case-control studies and one cohort study). In the case-control studies, clinical examination and National Institute of Neurologic and Communicative Disorders and Stroke- 
Alzheimer Disease and Related Disorders (NINCDS-ADRDA) criteria were dominant methods in the diagnosis of $\mathrm{AD}$ (respectively, $\mathrm{n}=11$ and $\mathrm{n}=7$ ). In the cohort studies, International Classification of Diseases (ICD) codes were most often used. Source of occupational exposure and jobs data were interview $(n=6)$, clinical records $(n=1)$, death certificate $(n=3)$, occupational records $(n=5)$, census $(n=2)$, interview with the next of kin $(n=3)$, a population-based study, and a telephone screening. Exposure assessment was based on the IH assessment, JEM, job title, and direct measurements. However, some studies used a mix of these methods for exposure assessment. Exposure creation for the studies except one (Savitz et al., 1998a) presented in $\mu \mathrm{T}$ (unit of magnetic flux density), was dominantly $0.2 \mu \mathrm{T}$. Additionally, some studies (Savitz et al., 1998b; Sorahan and Mohammed, 2014) used cumulative occupational exposure ( $\mu$ T-year) as their criteria. The risk estimates of studies (OR for case-control and RR, and HR for cohort studies) were presented for male and/or female and/or both genders.

Table 1. Characteristics of epidemiological studies on occupational exposure to ELF-MF and AD

\begin{tabular}{|c|c|c|c|c|c|c|c|}
\hline $\begin{array}{l}\text { First author, } \\
\text { Year } \\
\text { (Ref) }\end{array}$ & $\begin{array}{l}\text { Country } \\
\text { Time period } \\
\text { Number of } \\
\text { cases }\end{array}$ & $\begin{array}{l}\text { Study } \\
\text { design }\end{array}$ & $\begin{array}{l}\text { Outcome: } \\
\text { Source } \\
\text { Assessment } \\
\text { criteria }\end{array}$ & $\begin{array}{l}\text { Exposure: } \\
\text { Source } \\
\text { Assessment } \\
\text { criteria }\end{array}$ & Covariates & $\begin{array}{l}\text { Results }^{\dagger} \\
\text { - Male } \\
\text { - Female } \\
\text { - Both }\end{array}$ & $\begin{array}{l}\text { Bias } \\
\text { Score** }\end{array}$ \\
\hline $\begin{array}{l}\text { Sobel, } \\
1995 \# 1 \\
\text { (Sobel et al., } \\
1995)\end{array}$ & $\begin{array}{l}\text { - 1982-1985 } \\
\text { - Finland } \\
\text { - } 53\end{array}$ & Case-control & $\begin{array}{l}\text { - Medical records } \\
\text { - Clinical examination } \\
\text { - NINCDS-ADRDA }\end{array}$ & $\begin{array}{l}\text { - Interview } \\
\text { - } \mathrm{IH}^{\mathrm{b}} \text { assessment, Job } \\
\text { title, Direct } \\
\text { measurements } \\
\bullet>0.2 \mu \mathrm{T}\end{array}$ & $\begin{array}{l}\text { Gender, age at } \\
\text { examination, age at } \\
\text { onset, education }\end{array}$ & $\begin{array}{l}\cdot 0.70(0.10,8.90)^{\mathrm{c}} \\
-10.20(1.10,95.30) \\
\cdot 2.70(0.60,12.10)\end{array}$ & 9 \\
\hline $\begin{array}{l}\text { Sobel, } \\
1995 \# 2 \\
\text { (Sobel et al., } \\
1995)\end{array}$ & $\begin{array}{l}-1977-1978 \\
\text { - Finland } \\
\text { - } 198\end{array}$ & Case-control & $\begin{array}{l}\text { - Medical records } \\
\text { - Clinical examination } \\
\text { - NINCDS-ADRDA }\end{array}$ & $\begin{array}{l}\text { - Interview } \\
\text { - IH assessment, Job } \\
\text { title, Direct } \\
\text { measurements } \\
\bullet>0.2 \mu \mathrm{T}\end{array}$ & $\begin{array}{l}\text { Gender, age at } \\
\text { examination, social class }\end{array}$ & $\begin{array}{l}\text { - } 2.70(0.70,9.80) \\
\bullet 3.50(1.30,9.60) \\
\text { - } 3.20(1.50,7.20)\end{array}$ & 9 \\
\hline
\end{tabular}




\begin{tabular}{|c|c|c|c|c|c|c|c|}
\hline $\begin{array}{l}\text { Sobel, } \\
1995 \# 3 \\
\text { (Sobel et al., } \\
1995 \text { ) }\end{array}$ & $\begin{array}{l}\cdot 1984-1993 \\
\bullet \text { USA } \\
\bullet 136\end{array}$ & Case-control & $\begin{array}{l}\text { - Medical records } \\
\text { - Clinical examination } \\
\text { - NINCDS-ADRDA }\end{array}$ & $\begin{array}{l}\text { - Interview } \\
\text { - IH assessment, Job } \\
\text { title, Direct } \\
\text { measurements } \\
\bullet>0.2 \mu \mathrm{T}\end{array}$ & $\begin{array}{l}\text { Gender, age at } \\
\text { examination, education }\end{array}$ & $\begin{array}{l}\text { - } 1.70(0.30,10.30) \\
\text { - } 3.70(0.40,33.60) \\
\text { - } 2.40(0.60,9.20)\end{array}$ & 9 \\
\hline $\begin{array}{l}\text { Sobel, } \\
1996 \\
\text { (Sobel et al., } \\
1996)\end{array}$ & $\begin{array}{l}\cdot \mathrm{NR}^{\mathrm{d}} \\
\cdot \mathrm{USA} \\
\cdot 326\end{array}$ & case-control & $\begin{array}{l}\text { - Medical records } \\
\text { - Clinical examination } \\
\text { - NINCDS-ADRDA } \\
\text { (Probable or definite } \\
\text { AD) }\end{array}$ & $\begin{array}{l}\text { - Clinical records, } \\
\text { - IH assessment, } \\
\text { Direct } \\
\text { measurement, } \\
->0.2 \mu \mathrm{T}\end{array}$ & $\begin{array}{l}\text { Gender, age at } \\
\text { examination, education, } \\
\text { age at onset }\end{array}$ & $\begin{array}{l}\text { - } 4.90(1.34,7.89) \\
\text { - } 3.40(0.76,15.98) \\
\text { - } 3.93(1.45,10.56)\end{array}$ & 9 \\
\hline $\begin{array}{l}\text { Feychting, } \\
1998 \\
\text { (Feychting et } \\
\text { al., 1998) }\end{array}$ & $\begin{array}{l}\text { - 1989-1991 } \\
\text { - Sweden } \\
\text { - } 77\end{array}$ & case-control & $\begin{array}{l}\text { - A register-based } \\
\text { sample of twins } \\
\text { - Clinical examination } \\
\text { - NINCDS-ADRDA } \\
\text { (probable, possible } \\
\text { and definite AD) }\end{array}$ & $\begin{array}{l}\text { - Interview } \\
\text { - IH assessment, } \\
\mathrm{JEM}^{\mathrm{e}} \\
\bullet \geq 0.2 \mu \mathrm{T}\end{array}$ & Age, gender, education & $\begin{array}{l}\cdot- \\
\bullet- \\
\bullet 2.70(0.90,7.80)^{\mathrm{f}}\end{array}$ & 12 \\
\hline $\begin{array}{l}\text { Savitz, } \\
1998 \text { a } \\
\text { (Savitz et al., } \\
\text { 1998a) }\end{array}$ & $\begin{array}{l}\cdot \text { - 1985-1991 } \\
\bullet \text { USA } \\
\cdot 256\end{array}$ & Case-control & $\begin{array}{l}\text { - Death certificates } \\
\text { - Underlying cause of } \\
\text { death } \\
\text { - ICD }{ }^{\mathrm{g}}-9\end{array}$ & $\begin{array}{l}\text { - Death certificates } \\
\text { - Job title }\end{array}$ & $\begin{array}{l}\text { Age, calendar year, } \\
\text { social class, work status, } \\
\text { race }\end{array}$ & $\begin{array}{l}\bullet 1.20(1.00,1.40) \\
\bullet- \\
\bullet-\end{array}$ & 7 \\
\hline $\begin{array}{l}\text { Savitz, } \\
1998 \text { b } \\
\text { (Savitz et al., } \\
\text { 1998b) }\end{array}$ & $\begin{array}{l}\cdot \text { - 1950-1986 } \\
\text { - USA } \\
\text { - } 80\end{array}$ & cohort & $\begin{array}{l}\text { - Death certificates } \\
\text { - Underlying cause of } \\
\text { death } \\
\text { - ICD-9 }\end{array}$ & $\begin{array}{l}\text { - Occupational } \\
\text { records } \\
\text { - Direct } \\
\text { measurements } \\
\text { 2.35-14.5 } \mu \mathrm{T} \text {-year }\end{array}$ & $\begin{array}{l}\text { Age, calendar year, } \\
\text { social class, work status, } \\
\text { chemical exposures, race }\end{array}$ & $\begin{array}{l}\text { - } 2.70(0.80,8.90)^{\mathrm{h}} \\
\bullet- \\
\bullet-\end{array}$ & 11 \\
\hline $\begin{array}{l}\text { Graves, } \\
1999 \\
\text { (Graves, A. } \\
\text { B. et al., } \\
\text { 1999) }\end{array}$ & $\begin{array}{l}\text { - 1987-NR } \\
\text { - USA } \\
\text { - } 89\end{array}$ & Case-control & $\begin{array}{l}\text { - Medical records } \\
\text { - Clinical examination } \\
\text { - NINCDS-ADRDA } \\
\text { (probable, possible } \\
\text { and definite cases) }\end{array}$ & $\begin{array}{l}\bullet \text { Interview } \\
\bullet \mathrm{IH} \text { assessment } \\
\bullet \geq 0.3 \mu \mathrm{T}\end{array}$ & Age, education & $\begin{array}{l}\cdot- \\
\bullet- \\
\bullet 0.95(0.27,2.43)^{\mathrm{i}}\end{array}$ & 10 \\
\hline $\begin{array}{l}\text { Noonan, } \\
2002 \\
\text { (Noonan et } \\
\text { al., 2002) }\end{array}$ & $\begin{array}{l}\cdot 1987-1996 \\
\text { - USA } \\
\text { - } 1556\end{array}$ & Case-control & $\begin{array}{l}\text { - Death certificates } \\
\text { - Any mention of AD } \\
\text { ICD-9 }\end{array}$ & $\begin{array}{l}\text { - Death certificates } \\
\text { - JEM } \\
\bullet \geq 0.3 \mu \mathrm{T}\end{array}$ & $\begin{array}{l}\text { Age, race, occupational } \\
\text { grouping }\end{array}$ & $\begin{array}{l}\cdot 1.01(0.68,1.49) \\
\bullet- \\
\bullet-\end{array}$ & 10 \\
\hline
\end{tabular}




\begin{tabular}{|c|c|c|c|c|c|c|c|}
\hline $\begin{array}{l}\text { Feychting, } \\
2003 \\
\text { (Feychting et } \\
\text { al., 2003) }\end{array}$ & $\begin{array}{l}\bullet 1981-1995 \\
\bullet \text { Sweden } \\
\text { - } 2000\end{array}$ & Cohort & $\begin{array}{l}\text { - Death certificates } \\
\text { - Underlying cause of } \\
\text { death } \\
\text { - ICD-9 }\end{array}$ & $\begin{array}{l}\bullet \text { Census } \\
\text { - JEM } \\
\bullet \geq 0.5 \mu \mathrm{T}\end{array}$ & $\begin{array}{l}\text { Age, socioeconomic } \\
\text { status }\end{array}$ & $\begin{array}{l}\bullet 1.30(1.00,1.70)^{\mathrm{j}} \\
\bullet 2.30(1.00,5.20)^{\mathrm{j}} \\
\bullet-\end{array}$ & 11 \\
\hline $\begin{array}{l}\text { Håkansson, } \\
2003 \\
\text { (Håkansson } \\
\text { et al., 2003) }\end{array}$ & $\begin{array}{l}-1985-1996 \\
\text { - Sweden } \\
\text { - } 40\end{array}$ & Cohort & $\begin{array}{l}\text { - Death certificates } \\
\text { - primary or } \\
\text { contributing cause of } \\
\text { death } \\
\text { - ICD-8 and ICD-9 }\end{array}$ & $\begin{array}{l}\bullet \text { Census } \\
\bullet \text { JEM } \\
\bullet>0.53 \mu \mathrm{T}\end{array}$ & $\begin{array}{l}\text { Age, socioeconomic } \\
\text { status, gender }\end{array}$ & $\begin{array}{l}\cdot 2.70(0.88,8.25)^{\mathrm{k}} \\
\text { - } 22.70(1.32,390.82)^{\mathrm{k}} \\
\text { - } 4.04(1.40,11.66)^{\mathrm{k}}\end{array}$ & 12 \\
\hline $\begin{array}{l}\text { Harmanci, } \\
2003 \\
\text { (Harmanci et } \\
\text { al., 2003) }\end{array}$ & $\begin{array}{l}\bullet \text { NR } \\
\text { - Turkey } \\
\bullet 57\end{array}$ & Case-control & $\begin{array}{l}\text { - A population-based } \\
\text { study } \\
\text { - Clinical examination } \\
\text { - } \text { MMSE }^{\mathrm{l}} \text { and } \\
\mathrm{DSMMD}^{\mathrm{m}} \\
\text { - (Probable AD) }\end{array}$ & $\begin{array}{l}\text { - Interview with next } \\
\text { of kin } \\
\text { - IH assessment } \\
\bullet>0.2 \mu \mathrm{T}\end{array}$ & $\begin{array}{l}\text { Age, gender, education, } \\
\text { rural or urban, residence, } \\
\text { electrical } \\
\text { appliances, water } \\
\text { heating, medical history, } \\
\text { use of drugs, use of } \\
\text { alcohol }\end{array}$ & $\begin{array}{l}\cdot- \\
\bullet 4.02(1.02,15.78) \\
\bullet\end{array}$ & 11 \\
\hline $\begin{array}{l}\text { Qiu, } \\
2004 \\
\text { (Qiu et al., } \\
2004 \text { ) }\end{array}$ & $\begin{array}{l}\text { - } 1987-1996 \\
\text { - Sweden } \\
\text { - } 202\end{array}$ & Case-control & $\begin{array}{l}\text { - Medical records } \\
\text { - Clinical examination } \\
\text { DSMMD }\end{array}$ & $\begin{array}{l}\text { - Interview with } \\
\text { next of kin } \\
\text { - IH assessment, } \\
\text { JEM, direct } \\
\text { measurements } \\
\text { - } \geq 0.2 \mu \mathrm{T}\end{array}$ & $\begin{array}{l}\text { Age, education, } \\
\text { vascular disease, } \\
\text { Apolipoprotein, } \\
\text { E genotype, use of } \\
\text { alcohol, smoking, } \\
\text { mental activity, social } \\
\text { activity, gender }\end{array}$ & $\begin{array}{l}\text { - } 2.30(1.00,5.10)^{\mathrm{n}} \\
\text { - } 0.80(0.50,1.10)^{\mathrm{n}} \\
\text { - } 0.90(0.70,1.30)^{\mathrm{n}}\end{array}$ & 12 \\
\hline $\begin{array}{l}\text { Park, } \\
2005 \\
\text { (Park et al., } \\
2005 \text { ) }\end{array}$ & $\begin{array}{l}\cdot \text { 1992-1998 } \\
\text { - USA } \\
\text { - } 45\end{array}$ & Case-control & $\begin{array}{l}\text { - Death certificates } \\
\text { - Any mention of AD } \\
\text { - ICD-9 }\end{array}$ & $\begin{array}{l}\text { - Death certificates } \\
\text { - IH assessment, } \\
\text { JEM } \\
\bullet \geq 0.9 \mu \mathrm{T}\end{array}$ & $\begin{array}{l}\text { Age, race, gender, } \\
\text { region and } \\
\text { Socioeconomic status }\end{array}$ & $\begin{array}{l}\bullet- \\
\bullet- \\
\bullet 1.12(1.05,1.22)\end{array}$ & 12 \\
\hline $\begin{array}{l}\text { Davanipiour, } \\
2007 \\
\text { (Davanipour } \\
\text { et al., 2007) }\end{array}$ & $\begin{array}{l}\text { - Through } \\
1998 \\
\text { - USA } \\
\text { - } 1502\end{array}$ & Case-control & $\begin{array}{l}\text { - Medical records } \\
\text { - Clinical examination } \\
\text { - NINCDS-ADRDA } \\
\text { (probable or definite } \\
\text { AD) }\end{array}$ & $\begin{array}{l}\text { - occupational } \\
\text { records } \\
\text { - JEM } \\
\bullet>0.2 \mu \mathrm{T}\end{array}$ & $\begin{array}{l}\text { Gender, stroke, } \\
\text { smoking, income, } \\
\text { education, ethnicity, age } \\
\text { at examination or age at } \\
\text { onset }\end{array}$ & $\begin{array}{l}\text { - } 1.40(0.30,1.60) \\
\text { - } 3.30(1.30,8.40) \\
\text { - } 2.20(1.20,3.90)\end{array}$ & 10 \\
\hline
\end{tabular}




\begin{tabular}{|c|c|c|c|c|c|c|c|}
\hline $\begin{array}{l}\text { Roosli, } \\
2007 \\
\text { (Roosli et al., } \\
2007 \text { ) }\end{array}$ & $\begin{array}{l}\text { - } 1972-2002 \\
\text { - Switzerlan } \\
\text { d } \\
\text { - } 25\end{array}$ & Cohort & $\begin{array}{l}\text { - death certificates } \\
\text { - causes of death } \\
\text { - ICD-10 } \\
\text { - }\end{array}$ & $\begin{array}{l}\text { - Occupational } \\
\text { records } \\
\text { - Direct } \\
\text { measurements and } \\
\text { modelling of past } \\
\text { exposure } \\
\bullet>5.7 \mu \mathrm{T}\end{array}$ & Age, time period & $\begin{array}{l}\cdot 2.56(1.12,5.82) \\
\bullet- \\
\bullet-\end{array}$ & 12 \\
\hline $\begin{array}{l}\text { Seidler, } \\
2007 \\
\text { (Seidler, A. } \\
\text { et al., 2007) }\end{array}$ & $\begin{array}{l}\bullet \mathrm{NR} \\
\bullet \text { Germany } \\
\bullet 108\end{array}$ & Case-control & $\begin{array}{l}\text { - Medical records } \\
\text { - Clinical examination } \\
\text { - MMSE, HIS } \\
\text {, expert assessment } \\
\text { (possible AD) }\end{array}$ & $\begin{array}{l}\text { - Interview with the } \\
\text { next of kin } \\
\text { - IH assessment, } \\
\text { JEM, direct } \\
\text { measurements } \\
\text { - }>1 \mu \mathrm{T}\end{array}$ & $\begin{array}{l}\text { Age, region, gender, } \\
\text { dementia in parents, } \\
\text { smoking }\end{array}$ & $\begin{array}{l}\cdot \bullet- \\
\bullet- \\
\bullet 2.10(0.20,23.60)\end{array}$ & 10 \\
\hline $\begin{array}{l}\text { Andel, } \\
2010 \\
\text { (Andel et al., } \\
\text { 2010) }\end{array}$ & $\begin{array}{l}\text { - NR-1998 } \\
\text { - Sweden } \\
\text { - } 148\end{array}$ & cohort & $\begin{array}{l}\text { - A population-based } \\
\text { study, } \\
\text { - Clinical examination } \\
\text { NINCDS/ADRDA } \\
\text { (possible AD) }\end{array}$ & $\begin{array}{l}\text { - Telephone } \\
\text { screening, } \\
\text { - JEM } \\
\bullet \geq 0.2 \mu \mathrm{T}\end{array}$ & $\begin{array}{l}\text { Age, gender, education, } \\
\text { vascular risk factors }\end{array}$ & $\begin{array}{l}\cdot 1.80(0.64,5.05) \\
-1.08(0.57,2.07) \\
-1.38(0.88,2.26) \\
\text { - }\end{array}$ & 12 \\
\hline $\begin{array}{l}\text { Sorahan, } \\
2014 \\
\text { (Sorahan and } \\
\text { Mohammed, } \\
2014 \text { ) }\end{array}$ & $\begin{array}{l}\cdot 1973-2010 \\
\bullet \text { UK } \\
\bullet 170\end{array}$ & cohort & $\begin{array}{l}\text { - Death certificates } \\
\text { - Any mentioned of } \\
\text { AD } \\
\text { - ICD-9, ICD-10 }\end{array}$ & $\begin{array}{l}\text { - occupational } \\
\text { records } \\
\text { - JEM } \\
\text { - }>20 \mu \mathrm{T} \text {-year }\end{array}$ & $\begin{array}{l}\text { Gender, attained age, } \\
\text { calendar period and } \\
\text { socioeconomic status }\end{array}$ & $\begin{array}{l}\cdot- \\
\bullet- \\
\bullet 0.73(0.33,1.61)\end{array}$ & 12 \\
\hline $\begin{array}{l}\text { Davanipiour, } \\
2014 \\
\text { (Davanipour } \\
\text { et al., 2014) }\end{array}$ & $\begin{array}{l}\cdot \text { 1993-1994 } \\
\text { - USA } \\
\text { - } 45\end{array}$ & case-control & $\begin{array}{l}\text { - A Population-based } \\
\text { study } \\
\text { - Clinical examination } \\
\text { - MMSE (dementia } \\
\text { including two-thirds } \\
\text { of AD cases) }\end{array}$ & $\begin{array}{l}\text { - Interview, } \\
\text { - IH assessment, } \\
\text { JEM } \\
\bullet>1 \mu \mathrm{T}\end{array}$ & $\begin{array}{l}\text { Gender, age, education, } \\
\text { family income, stroke, } \\
\text { smoking, alcohol } \\
\text { consumption. }\end{array}$ & $\begin{array}{l}\cdot- \\
\bullet- \\
\bullet 3.40(1.30,8.90)^{\mathrm{c}}\end{array}$ & 11 \\
\hline $\begin{array}{l}\text { Koeman, } \\
2015 \\
\text { (Koeman et } \\
\text { al., 2015) }\end{array}$ & $\begin{array}{l}\text { - 1986-2003 } \\
\text { - Netherland } \\
\text { s } \\
\text { - } 232\end{array}$ & Cohort & $\begin{array}{l}\text { - Death certificates } \\
\text { - Causes of death } \\
\text { - ICD-9, ICD-10 }\end{array}$ & $\begin{array}{l}\text { - A population-based } \\
\text { study } \\
\text { - JEM } \\
\text { - }>0.3 \mu \mathrm{T}\end{array}$ & $\begin{array}{l}\text { Smoking status, physical } \\
\text { activity, body mass } \\
\text { index }\end{array}$ & $\begin{array}{l}\text { - } 0.91(0.39,2.12) \\
\text { - - } \\
\bullet-\end{array}$ & 12 \\
\hline
\end{tabular}




\begin{tabular}{|l|l|l|l|l|l|l|}
\hline Pedersen, & $\bullet 1982-2010$ & Cohort & $\bullet$ Medical records & $\bullet$ Occupational & Gender, age, calendar & $\bullet 1.13(0.70,1.82)$ \\
2017 & $\bullet$ Denmark & & $\bullet$ Clinical examination & records & year, electric shock, job & $\bullet-$ \\
(Pedersen et & $\bullet 17$ & & $\bullet$ ICD-8, ICD-10 & $\bullet$ JEM & \\
al., 2017) & & & & $\bullet \geq 1 \mu \mathrm{T}$ & \\
\hline
\end{tabular}

${ }^{+}$risk estimates (odds ratio for case-control studies and relative risk for cohort studies) and $95 \%$ confidence interval; $*$ combined male and female results; ** zero score indicates the highest risk of bias and twelve score indicates the lowest risk of bias; ${ }^{a}$ criteria from the National Institute of Neurologic and Communicative Disorders and Stroke-Alzheimer Disease and Related Disorders Association; ${ }^{\mathrm{b}}$ Industrial Hygienist; ${ }^{\mathrm{c}}$ Unadjusted;

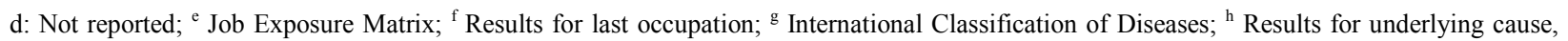
with exposure $\geq 20 \mu \mathrm{T}$-year; ${ }^{\mathrm{i}}$ Results for ever/never exposed- Industrial hygienist $2 ;{ }^{j}$ Results for total occupation in $1970 ;{ }^{\mathrm{k}}$ Results for primary or contributing cause of death; ${ }^{1}$ Mini Mental State Examination; ${ }^{\mathrm{m}}$ criteria from the Diagnostic and Statistical Manual of Mental Disorders; ${ }^{\mathrm{n}}$ Results for exposure in lifetime principal occupation; ${ }^{\circ}$ Hachinski Ischaemic Score; p: MMSE score $<10$

\subsection{Primary meta-analysis results}

Figure 2 shows forest plot of studies with considering occupational exposure to ELF-MF and risk of $\mathrm{AD}$. 


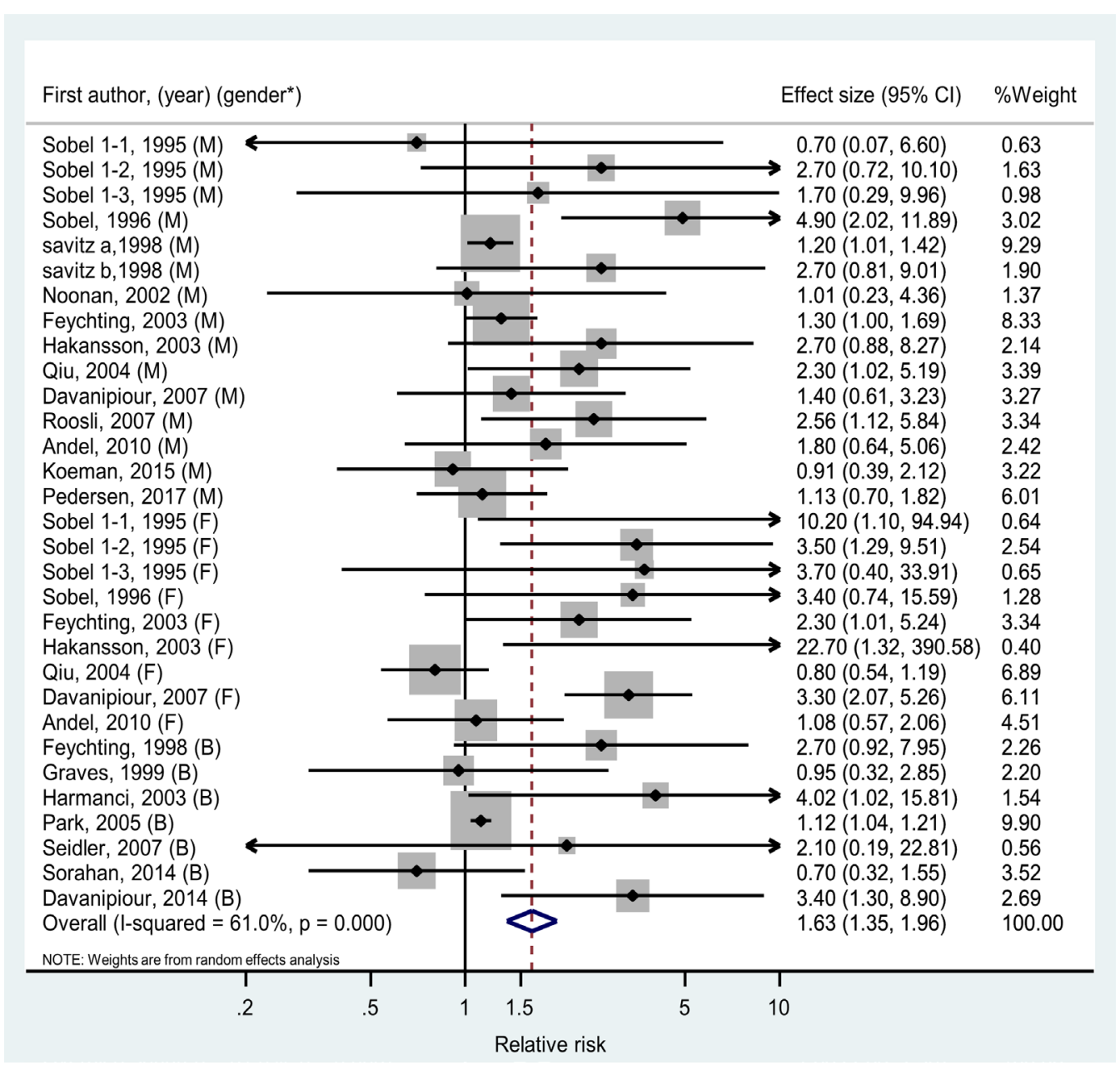

*M: Male; F: Female; B: Both genders combined

Figure 2. Forest plot of studies on the risk of AD and occupational ELF-MF exposure. Risk estimates and $95 \%$ confidence intervals (CI) for the individual studies are depicted as squares and horizontal lines, respectively. Pooled estimate with $95 \% \mathrm{CI}$ are depicted as open diamonds The results displayed in the Figure 2 show moderate to high heterogeneity amongst studies $\left(\mathrm{I}^{2}=61.0 \%, \mathrm{p}<0.001\right)$. In addition, the pooled of 31 risk estimates was obtained $(\mathrm{RR}=1.63 ; 95 \%$ CI: $1.35,1.96)$. Therefore, ELF-MF exposed workers have a 1.63 times increased risk to develop $\mathrm{AD}$ (Figure 2).

\subsection{Risk of bias within studies}


We found that Savitz study has the highest risk of bias (7 stars). Totally, 17 out of 22 studies showed a low risk of bias ( $\geq 10$ stars) (Table 1 and supplementary table S2). Figure 3 shows Sensitivity of the effect estimate when leaving out sets of studies based on the risk of bias.

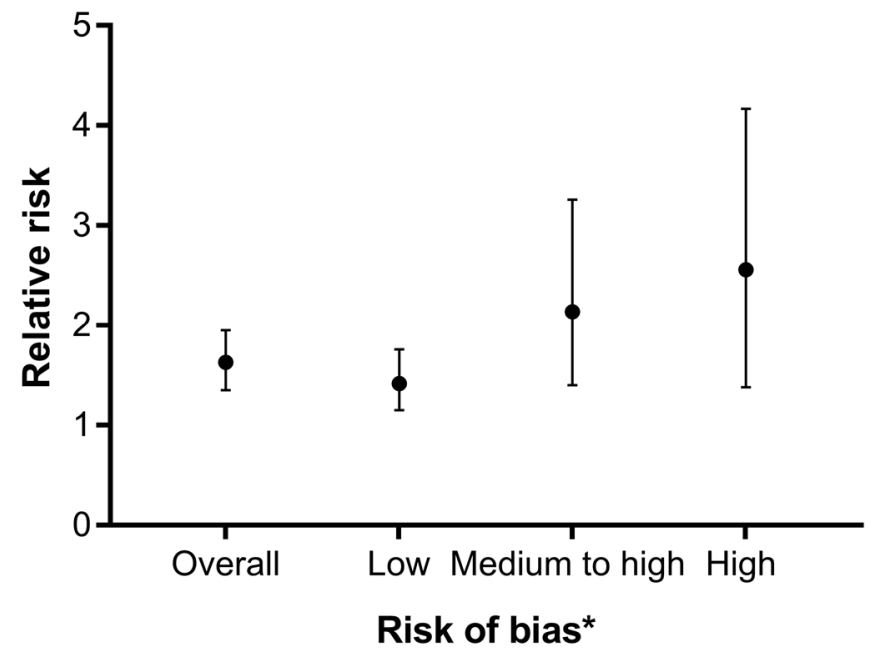

*Overall: the mean pooled estimate; Low: pooled estimate of studies with score of $>10$; Medium to high: pooled estimate of studies with score of $\leq 10$; High: pooled estimate of studies with score of $<10$

Figure 3. Sensitivity of the effect estimate when leaving out sets of studies based on bias score Results of Figure 2 indicated the pooled estimate of 1.42 (95\% CI: 1.15, 1.76), 2.14 (95\% CI: $1.40,3.26)$, and $2.56(95 \% \mathrm{CI}: 1.38,4.17)$ for studies with low, medium to high, and high risk of bias, respectively.

These findings revealed that risk of bias was higher in the studies with the higher pooled estimate. However, overall pooled estimate and heterogeneity values were slightly higher in compared to the pooled estimate of studies with low risk of bias.

\subsection{Subgroup analysis}

Figure 4 shows the forest plot of some jobs which were exposed to ELF-MF and risk of AD. 


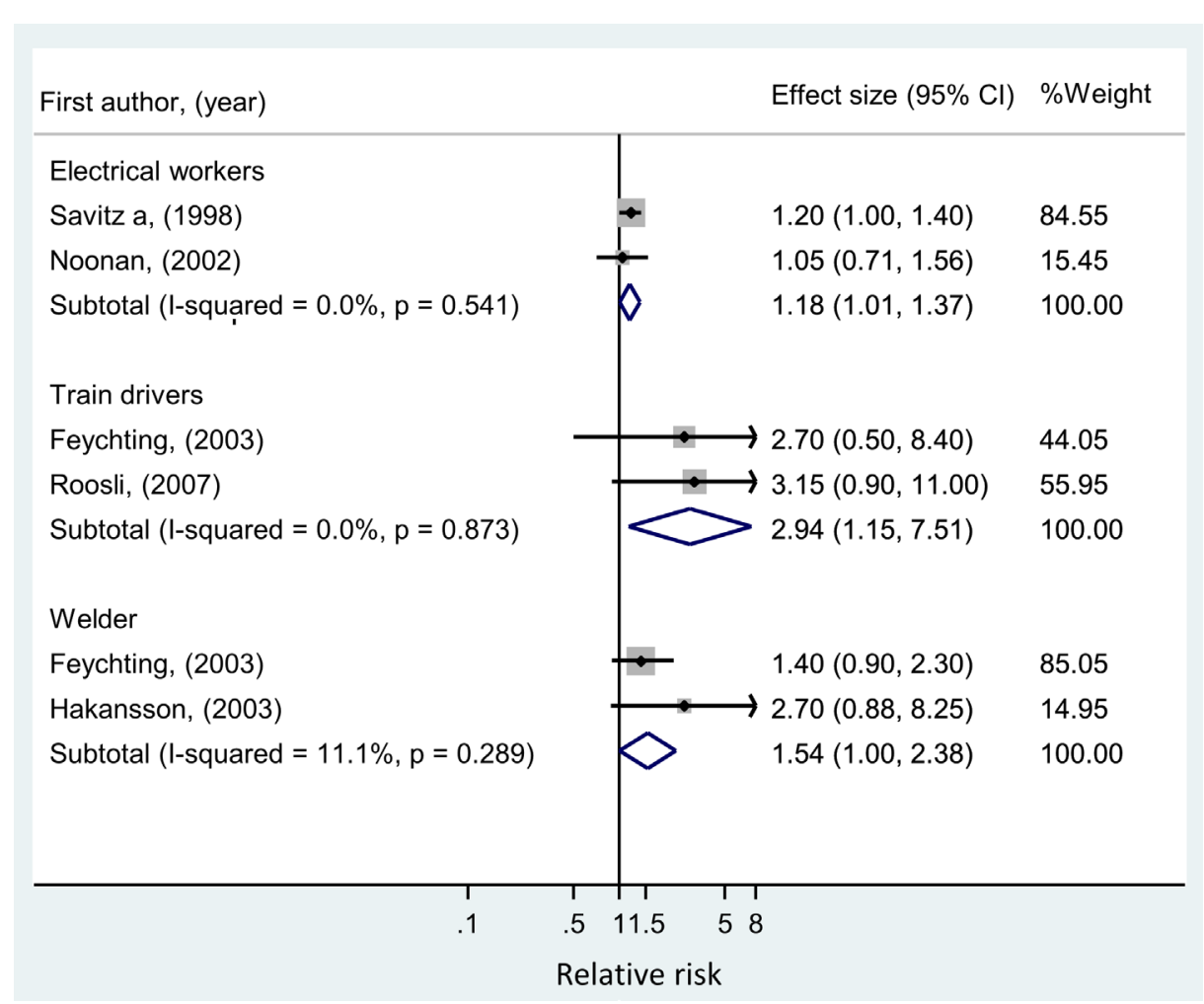

Figure 4. Forest plot of studies on some jobs. Risk estimates and 95\% confidence intervals (CI) for individual studies are depicted as squares and horizontal lines, respectively. Pooled estimates with $95 \% \mathrm{CI}$ are depicted as open diamonds

The results of subgroup analyses showed that train drivers had a highest risk of $\mathrm{AD}(\mathrm{RR}=2.94$; $95 \%$ CI: $1.15,7.51)$ than electrical workers and welders. However, the differences among three jobs were not statistically significant $(\mathrm{Q}=4.74, \mathrm{df}=2, \mathrm{p}=0.094)$ (Figure 4$)$.

Table 2. Subgroup meta-analysis to assess the effect of some risk factors on AD

\begin{tabular}{|c|c|c|c|c|c|c|}
\hline Characteristics & Factors & Sample $^{\mathrm{a}}$ & $\mathrm{RR}(95 \% \mathrm{CI})$ & I-Square (\%) & $\mathrm{P}^{\mathrm{b}}{ }_{\text {heterogeneity }}$ & $\mathrm{P}^{\mathrm{c}}{ }_{\text {meta-regression }}$ \\
\hline \multirow{3}{*}{ Gender } & Male & 15 & $1.50(1.22,1.85)$ & 30.0 & \multirow{3}{*}{$<0.001$} & \multirow{3}{*}{0.43} \\
\hline & Female & 9 & $2.39(1.29,4.40)$ & 75.8 & & \\
\hline & Both & 7 & $1.50(0.94,2.41)$ & 52.6 & & \\
\hline \multirow{3}{*}{ Country } & USA & 12 & $1.77(1.33,2.38)$ & 72.7 & \multirow{3}{*}{$<0.001$} & \multirow{3}{*}{0.67} \\
\hline & Nordic countries ${ }^{\mathrm{d}}$ & 14 & $1.63(1.21,2.20)$ & 50.5 & & \\
\hline & Other $^{\mathrm{e}}$ & 5 & $1.48(0.74,2.93)$ & 52.2 & & \\
\hline
\end{tabular}




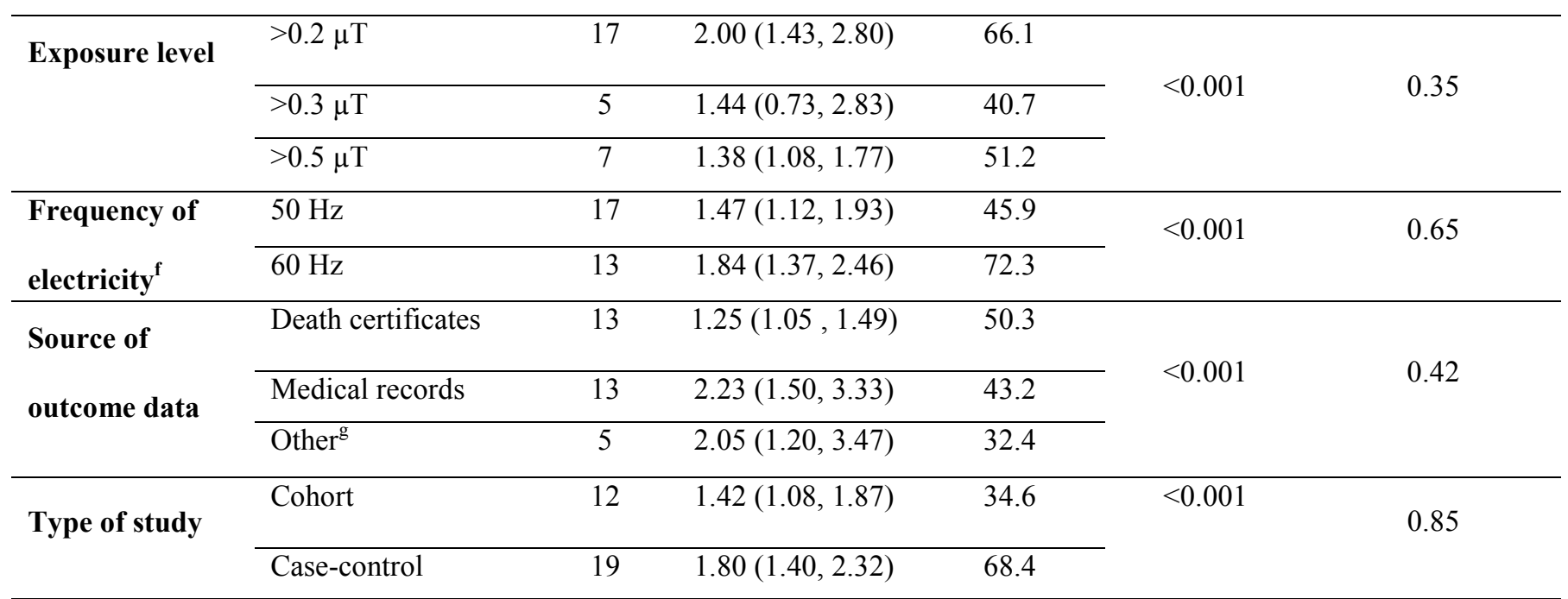

a (See supplementary Table S3); ${ }^{\mathrm{b}}$ P-value for subgroup meta-analysis; ${ }^{\mathrm{c}}$ P-value for meta-regression; ${ }^{\mathrm{d}}$ Include Denmark, Sweden, and Finland; ' ${ }^{\mathrm{e}}$ Including Turkey, German, the Netherlands, Switzerland, and the UK; ${ }^{\mathrm{f}} 50 \mathrm{~Hz}$ is frequency of electricity in the Europe and $60 \mathrm{~Hz}$ is the frequency of electricity in the USA and Asia; ${ }^{\mathrm{g}}$ Including hospital records, population studies, and register-based database

According to the results of nine risk estimates, the risk of $\mathrm{AD}$ for female workers $(\mathrm{RR}=2.39$; 95\% CI: $1.29,4.40)$ was significantly higher than males. Estimates differed significantly between countries with pooled estimate of 1.77 (95\% CI: 1.33, 2.38), 1.63 (95\% CI: 1.21, 2.20), and 1.48 (95\% CI: $0.74,2.93)$ for studies conducted in the USA, Nordic countries, and other countries, respectively. Subgroup analysis according to the cut-off point used to differentiate between the exposed and non-exposed subjects revealed a higher risk of $\mathrm{AD}$ for $>0.2 \mu \mathrm{T}(\mathrm{RR}=2.00$; $95 \% \mathrm{CI}$ : $1.43,2.80)$ compared to $>0.3$ or $0.5 \mu \mathrm{T}$. A significant difference $(\mathrm{p}<0.001)$ was shown between the risk of $\mathrm{AD}$ for different frequencies of electricity $(50 \mathrm{~Hz}$ vs. $60 \mathrm{~Hz})$. In terms of the source of outcome data, the risk of $\mathrm{AD}$ among studies with medical records $(\mathrm{RR}=2.23 ; 95 \% \mathrm{CI}: 1.50,3.33)$ was significantly higher than other sources of outcome data $(\mathrm{p}<0.001)$. The risk of AD was found to be higher in the case-control than in cohort studies $(\mathrm{p}<0.001)$.

The results of meta-regression showed that none of covariates were the sources of heterogeneity $(\mathrm{P}>0.1)$. 
The results of Figure S2 (supplementary file) indicated a relatively stable and weak association (RR: $1.21 ; 95 \%$ CI: $1.13,1.28)$ between exposure to ELF-MF and AD risk.

\subsection{Publication bias}

Publication bias was assessed using both funnel plot (Figure 5) and Egger's test (31 risk estimates, case-control and cohort studies). According to the funnel plot, considerable asymmetry indication publication bias for studies on the risk of AD and ELF-MF exposure.

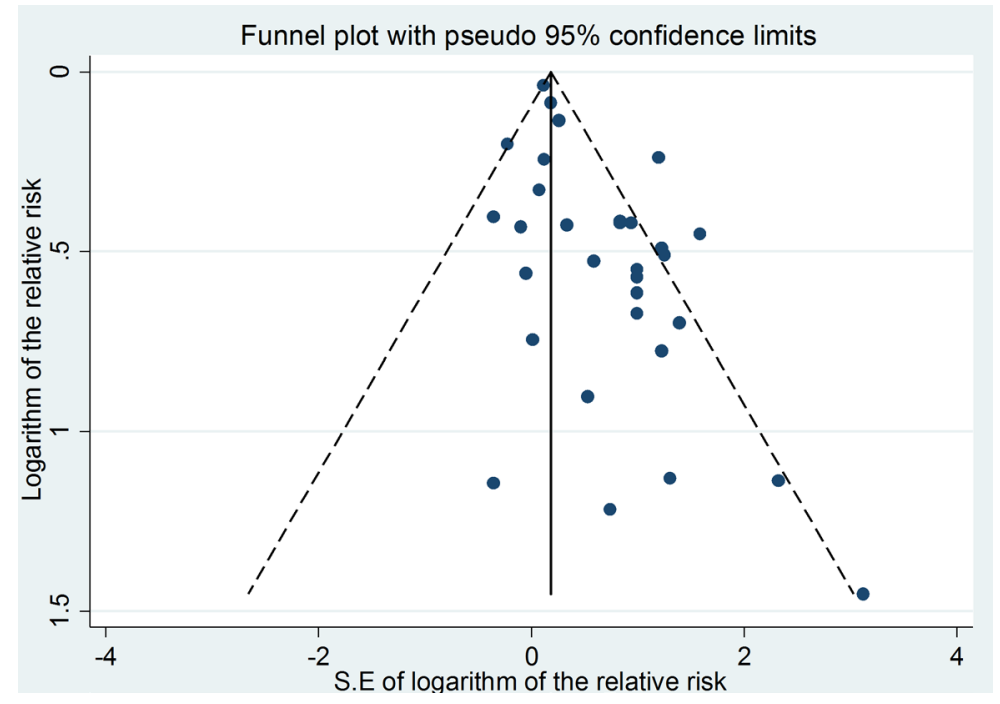

Figure 5. Funnel plot for all individual studies. Each points represents a separate study.

Egger's test showed indications of publication bias (Intercept $=1.18 ; 95 \%$ CI: 0.60, 1.75; $\mathrm{p}<0.001)$. Nevertheless, after applying the fill-and-trim method, the adjusted pooled estimate of the risk of $\mathrm{AD}$ was similar $(\mathrm{RR}=1.63 ; 95 \% \mathrm{CI}: 1.35,1.96)$ to the unadjusted pooled estimate, as shown in Figure 2.

\section{Discussion}

We conducted a systematic review and meta-analysis of twenty-two epidemiological studies (20 articles) on the association between occupational exposure to ELF-MF and the risk of AD. The results revealed a significant 1.63 fold increase in the risk of AD among ELF-MF-related occupations with some levels of heterogeneity across gender, country, exposure level, and source 
of outcome data, but not with respect to the job title. In addition, there was an indication for publication bias; however, by adjusting for publication bias, no change was observed in the pooled estimate.

Some studies have explored this relationship (García et al., 2008; Vergara et al., 2013). Similar risk estimates have been reported by meta-analyses of Garcia et al. (2008) and Huss et al. (2014) (García et al., 2008; Huss and Vermeulen, 2014). However, the Huss et al. study is just a part of a book and they have no report on subgroups. But Vergara et al. found a small association (RR: 1.27; 95\% CI: 1.15, 1.40) between ELF-MF exposure and AD risk (Vergara et al., 2013) and it looks underestimate the risk. Based on these comparisons, it seems they have chosen wrong studies or effect sizes. For example, they have excluded study of Harmanci et al., a will done study with no risk of bias, and have included two studies (Salib and Hillier, 1996; Stampfer, M. J., 2009) with no propose to assess ELF-MF exposure and AD risk in the final analysis.

This is true that incidence of $\mathrm{AD}$ is more in women (75-94 years old, 24.25\%) compered to men (75-95 years old, 17.9\%) (Vina and Lloret, 2010) and fact can explain why, in the current findings, the pooled estimate for women is more than men.

Although death certificate is considered to be a valuable source for epidemiological studies, dementia is underreported as primary and contributory causes of death (Garcia-Ptacek et al., 2016). The current systematic review showed that five (Feychting et al., 2003; Håkansson et al., 2003; Koeman et al., 2015; Roosli et al., 2007; Savitz et al., 1998b) out of eight cohort studies (62\%) considered the cause of death as the assessment method for AD. In addition, there are diagnostic difficulties in accurately identifying and distinguishing $\mathrm{AD}$ and vascular dementia ( $\mathrm{T}$ O'Brien and Thomas, 2015). Likewise, no code was specifically defined for AD in the ICD-8, and there were two (Håkansson et al., 2003; Pedersen et al., 2017) studies that used this 
classification. Therefore, underestimation and misclassification of $\mathrm{AD}$ is to be expected in death certificate-based studies where cases are no longer available for detection or re-assessment. Such misclassification is unlikely to be related to ELF-MF exposure, and is thus expected to result in a bias to unity if there is a real association. In contrast, $57 \%$ of the case-control studies used medical records, a more reliable data source (Johansson et al., 2009), mostly clinical examinations and NINCDS-ADRDA, a widely accepted criteria (Blacker et al., 1994), to diagnose AD (Davanipour et al., 2007; Feychting et al., 1998; Graves, A. B. et al., 1999; Sobel et al., 1995; Sobel et al., 1996). Nevertheless, while clinical diagnoses of AD may be preferable to death certificates, the gold standard diagnosis is examination of the brain upon autopsy. Thus, in principle one would expect less bias from these studies in comparison with death certificatesbased studies. However, in the Davanipiour et al. (Davanipour et al., 2014) and Pedersen et al.s' (Pedersen et al., 2017) studies, some cases were considered as AD cases, while they may have suffered from other types of dementia. In addition, in most case-control studies, possible and probable $\mathrm{AD}$ cases had participated in the research, and this might also be a source of bias. Again, most likely this is non-differential misclassification. For case-control studies, however, there was a potential for selection bias in the control selection (i.e. the controls occupationally/ residentially/environmentally have been exposed to ELF-MF), in particular in those studies selecting controls from hospitals or next of kin. Actually, different control selection strategies employed in different studies may be a source of variation. In addition, selection of cases from hospital records may be a source of bias, because these subjects may not be representative of the cases in the source population. Hospital records just reflect those subjects who were brought to hospitals or medical centers by their next of kin (usually his/her family members). If they were in denial, just in moderate-to-severe dementia the patients received medical attention (Borenstein 
and Mortimer, 2016). So that, these conditions may explain moderate to high level of heterogeneity in the case-control studies (Table 2, $\mathrm{I}^{2}: 68.4 \%$ ) rather than cohort studies (Table 2, $\left.\mathrm{I}^{2}: 34.6 \%\right)$. Consequently, no definite statement is possible whether cohort or case-control studies are less biased.

The most useful and reliable methods for occupational exposure assessment are direct measurements including spot measurement and personal exposure measurements. Personal measurements during the whole work are particularly useful for exposure assessment. In this method, a portable device (e.g. Standard EMDEX II) is attached to the worker and records the ELF-MF in a whole workday. For long term exposure assessment, full history of each occupation and all possible exposure situations should be considered (Röösli and Vienneau, 2014). Only six reports used measurement methods (four spot measurements and two personal measurements) to assess some jobs exposure (Qiu et al., 2004; Roosli et al., 2007; Savitz et al., 1998b; Seidler, A. et al., 2007; Sobel et al., 1995; Sobel et al., 1996). Direct methods are not feasible and costeffective for all studies; hence, alternative methods including IH assessment, JEM, and job title assessment had been used. The advantage of a JEM is the systematic approach, which does not introduce differential exposure misclassification. On the other hand, IH is more likely to be subjective. For instance, it seems that IH assessment of Seidler et al. for category of occupations had led to higher exposure of classification categories containing low exposure jobs. In this study, based on an expert rating, the maximum exposure of a white-collar worker to ELF-MF has been estimated $5.5 \mu \mathrm{T}$, but they considered $0.15 \mu \mathrm{T}$ for the maximum exposure of a dressmaker. In this misclassification, they categorized and analyzed the white-collar workers as a highexposed job, but the dressmakers were categorized in moderate to low exposure category for analysis (Seidler, A. et al., 2007). 
Covariates are one of the most important factors in estimating the relationship between occupational exposure to ELF-MF and the risk of AD. Previous studies suggested occupational risk factors including exposure to pesticides, welding fumes, metals such as aluminum, and polychlorinated biphenyls (PCBs) on $\mathrm{AD}$. In addition, personal characteristics and family history are other risk factors (Richardson et al., 2014). Therefore, controlling a wide range of confounders is hard and perhaps impossible, and they can be a source of error in these studies. For example, occupational exposure to PCBs was reported amongst utility workers (Charles et al., 2003). Likewise, occupational exposure to welding fumes was suggested as a risk factor for neurodegenerative diseases in welders (Stampfer, Meir J, 2009). Overall, just one study had considered chemical exposure, and it suggested a positive relationship between exposure to ELFMF and the increasing risk of AD (Savitz et al., 1998b).

In summary, bias from confounding, outcome and exposure misclassification is a concern given the high heterogeneity between the studies.

Biological plausibility of in vitro and in vivo studies suggests neuroinflammatory processes as the mechanism to explain ELF-EMF potential actions on AD (Grammas, 2011). The main theoretical premise behind this hypothesis is that exposure to ELF-MF can promote inflammation processes and thus influence the progression of neurodegenerative diseases including AD (Galasko and Montine, 2010; Grammas, 2011). However, a recent report suggests that long-term exposure (18 months) to ELF-MF $(50 \mathrm{~Hz}, 1 \mathrm{mT})$ has no effect on the cellular processes involved in the pathogenesis of AD (Liebl et al., 2015). Only few in vivo studies are available to support this theory; most recent studies such as Liebl et al. (2016) (Liebl et al., 2015) (the first comprehensive study on the association of ELF-MF with AD in mouse models) or Maes et al. (2016) (Maes et al., 2016) suggested that further investigations are needed. In 
general, experimental findings are based on short-term exposure to very high ELF-MF (typically

$\geq 1 \mathrm{mT}$ ) and these studies do not represent realistic occupational exposure scenarios (long-term at lower levels). In conclusion, heterogeneity regarding the ELF-MF intensity, the cell type, biological endpoint, and the time point of investigation are seen in these experimental studies (Mattsson and Simko, 2012).

Overall, the current study was limited to English- language, original articles, and this might have led to bias in this study. Studies with negative reports are more likely to be rejected, and eventually they are published in the form of non-English or gray articles. Moreover, like other meta-analysis papers, decision about variables made by authors may introduce some bias.

\section{Conclusion}

This systematic review and meta-analysis suggests that occupational exposure to ELF-MF may increase the risk of AD. However, this suggestion should be interpreted with caution given the moderate to high heterogeneity, and indication for publication bias. Finally, more studies are essential to gain a better understanding on the effects of ELF-MF with the risk of AD.

\section{Acknowledgment}

This work was supported by Shiraz University of Medical Sciences, Shiraz, Iran (grant NO. 9501-42-13500). The authors would like to thanks the Research Center for Health Sciences and Research Consultation Center (RCC) at Shiraz University of Medical Sciences for their invaluable assistance in editing this manuscript.

\section{References:}

2017. EMF-Portal, . Available online: http://www.emf-portal.de/. ACGIH, 2015. TLVs and BEIs. kemper meadow drive , Cincinnati , OH, 124-126. 
Ahlbom, I.C., Cardis, E., Green, A., Linet, M., Savitz, D., Swerdlow, A., Epidemiology, I.S.C.o., 2001. Review of the epidemiologic literature on EMF and Health. Environmental Health Perspectives 109(Suppl 6), 911-933.

Andel, R., Crowe, M., Feychting, M., Pedersen, N.L., Fratiglioni, L., Johansson, B., Gatz, M., 2010. Work-related exposure to extremely low-frequency magnetic fields and dementia: results from the population-based study of dementia in Swedish twins. The journals of gerontology. Series A, Biological sciences and medical sciences 65(11), 1220-1227.

Blacker, D., Albert, M.S., Bassett, S.S., Go, R.P., Harrell, L.E., Folstein, M.F., 1994. Reliability and validity of NINCDS-ADRDA criteria for alzheimer\&\#39;s disease: The national institute of mental health genetics initiative. Archives of Neurology 51(12), 1198-1204.

Borenstein, A.R., Mortimer, J.A., 2016. Chapter 9 - Introduction to the Analytic Epidemiology of Alzheimer's Disease, Alzheimer's Disease. Academic Press, San Diego, pp. 97-106.

Bowman, J.D., Touchstone, J.A., Yost, M.G., 2007. A population-based job exposure matrix for powerfrequency magnetic fields. Journal of occupational and environmental hygiene 4(9), 715-728.

Charles, L.E., Loomis, D., Shy, C.M., Newman, B., Millikan, R., Nylander-French, L.A., Couper, D., 2003. Electromagnetic fields, polychlorinated biphenyls, and prostate cancer mortality in electric utility workers. American journal of epidemiology 157(8), 683-691.

Davanipour, Z., Tseng, C.-C., Lee, P.-J., Markides, K.S., Sobel, E., 2014. Severe cognitive dysfunction and occupational extremely low frequency magnetic field exposure among elderly Mexican Americans. British journal of medicine and medical research 4(8), 1641.

Davanipour, Z., Tseng, C.C., Lee, P.J., Sobel, E., 2007. A case-control study of occupational magnetic field exposure and Alzheimer's disease: Results from the California Alzheimer's Disease Diagnosis and Treatment Centers. BMC Neurology 7.

Eskelinen, T., Roivainen, P., Mäkelä, P., Keinänen, J., Kauhanen, O., Saarikoski, S., Juutilainen, J., 2016. Maternal exposure to extremely low frequency magnetic fields: Association with time to pregnancy and foetal growth. Environment International 94, 620-625.

Feychting, M., Jonsson, F., Pedersen, N.L., Ahlbom, A., 2003. Occupational magnetic field exposure and neurodegenerative disease. Epidemiology 14(4), 413-419.

Feychting, M., Pedersen, N.L., Svedberg, P., Floderus, B., Gatz, M., 1998. Dementia and occupational exposure to magnetic fields. Scandinavian Journal of Work, Environment and Health 24(1), 46-53. Galasko, D., Montine, T.J., 2010. Biomarkers of oxidative damage and inflammation in Alzheimer's disease. Biomarkers in medicine 4(1), 27-36.

Garcia-Ptacek, S., Kåreholt, I., Cermakova, P., Rizzuto, D., Religa, D., Eriksdotter, M., 2016. Causes of Death According to Death Certificates in Individuals with Dementia: A Cohort from the Swedish Dementia Registry. Journal of the American Geriatrics Society 64(11), e137-e142.

García, A.M., Sisternas, A., Hoyos, S.P., 2008. Occupational exposure to extremely low frequency electric and magnetic fields and Alzheimer disease: a meta-analysis. International Journal of Epidemiology 37(2), 329-340.

Grammas, P., 2011. Neurovascular dysfunction, inflammation and endothelial activation: implications for the pathogenesis of Alzheimer's disease. J. Neuroinflammation 8, 26.

Graves, A.B., Rosner, D., Echeverria, D., Yost, M., Larson, E.B., 1999. Occupational exposure to electromagnetic fields and Alzheimer disease. Alzheimer Disease and Associated Disorders 13(3), 165170.

Graves, A.B., Rosner, D., Echeverria, D., Yost, M., Larson, E.B., 1999. Occupational exposure to electromagnetic fields and Alzheimer disease. Alzheimer Dis. Assoc. Disord. 13(3), 165-170.

Greenland, S., Sheppard, A.R., Kaune, W.T., Poole, C., Kelsh, M.A., 2000. A pooled analysis of magnetic fields, wire codes, and childhood leukemia. Childhood Leukemia-EMF Study Group. Epidemiology 11(6), 624-634.

Håkansson, N., Gustavsson, P., Johansen, C., Floderus, B., 2003. Neurodegenerative diseases in welders and other workers exposed to high levels of magnetic fields. Epidemiology 14(4), 420-426. 
Harmanci, H., Emre, M., Gurvit, H., Bilgic, B., Hanagasi, H., Gurol, E., Sahin, H., Tinaz, S., 2003. Risk factors for Alzheimer disease: A population-based case-control study in Istanbul, Turkey. Alzheimer Disease and Associated Disorders 17(3), 139-145.

Huss, A., Vermeulen, R., 2014. Neurodegenerative Diseases and ELF-EMF, in: Röösli, M. (Ed.)

Epidemiology of Electromagnetic Fields. CRC Press, Boca Raton, pp. 185-203.

ICNIRP, 2010. Guidelines for limiting exposure to time-varying electric and magnetic fields (1 $\mathrm{Hz}$ to 100 $\mathrm{kHz}$ ). Health physics 99(6), 818-836.

Jalilian, H., Monazzam, M.R., Zakerian, S.A., Zokaie, M., Najafi, K., 2016. Mental health status among workers exposed to extremely low frequency magnetic fields. Occupational Medicin Quarterly Journal 7(3), 57-68.

Jalilian, H., Najafi, K., Monazzam, M.R., Khosravi, Y., Zamanian, Z., 2017. Assessment of static and extremely low frequency magnetic fields in the electric-powered trains: An environmental and occupational perspective. International Journal of Occupational Hygiene 9(2).

Johansson, L.A., Bjorkenstam, C., Westerling, R., 2009. Unexplained differences between hospital and mortality data indicated mistakes in death certification: an investigation of 1,094 deaths in Sweden during 1995. Journal of clinical epidemiology 62(11), 1202-1209.

Kheifets, L., Monroe, J., Vergara, X., Mezei, G., Afifi, A.A., 2008. Occupational electromagnetic fields and leukemia and brain cancer: an update to two meta-analyses. Journal of occupational and environmental medicine 50(6), 677-688.

Koeman, T., Schouten, L.J., van den Brandt, P.A., Slottje, P., Huss, A., Peters, S., Kromhout, H., Vermeulen, R., 2015. Occupational exposures and risk of dementia-related mortality in the prospective Netherlands Cohort Study. American journal of industrial medicine 58(6), 625-635.

Liebl, M.P., Windschmitt, J., Besemer, A.S., Schafer, A.K., Reber, H., Behl, C., Clement, A.M., 2015. Low-frequency magnetic fields do not aggravate disease in mouse models of Alzheimer's disease and amyotrophic lateral sclerosis. Scientific reports 5,8585 .

Maes, A., Anthonissen, R., Wambacq, S., Simons, K., Verschaeve, L., 2016. The Cytome Assay as a Tool to Investigate the Possible Association Between Exposure to Extremely Low Frequency Magnetic Fields and an Increased Risk for Alzheimer's Disease. Journal of Alzheimer's disease : JAD 50(3), 741-749. Mattsson, M.O., Simko, M., 2012. Is there a relation between extremely low frequency magnetic field exposure, inflammation and neurodegenerative diseases? A review of in vivo and in vitro experimental evidence. Toxicology 301(1-3), 1-12.

McRobbie, D.W., 2012. Occupational exposure in MRI. The British Journal of Radiology 85(1012), 293312.

Monazzam, M., Jalilian, H., Najafi, K., zakerian, S., 2015. Single-Axis and Three-Axis Probe Magnetic Field Meters in an Occupational Hygiene Study: A Comparative View. International Journal of Occupational Hygiene 7(4), 166-171.

Monazzam, M.R., Jalilian, H., Khosravi, Y., Najafi, K., Zamanian, Z., 2016. Occupational Exposure Assessment of Tehran Metro Drivers to Extremely Low Frequency Magnetic Fields. Journal of Occupational Health Engineering 2(4), 58-64.

Monazzam, M.R., Jalilian, H., Najafi, K., Zakerian, S.A., Emkani, M., Hadadi, M., 2015. Environmental evaluation and employee's exposure of a thermal power plant with extremely low frequency magnetic fields. Iran Occupational Health Journal 12(3), 65-75.

Noonan, C.W., Reif, J.S., Yost, M., Touchstone, J., 2002. Occupational exposure to magnetic fields in case-referent studies of neurodegenerative diseases. Scandinavian Journal of Work, Environment and Health 28(1), 42-48.

Park, R.M., Schulte, P.A., Bowman, J.D., Walker, J.T., Bondy, S.C., Yost, M.G., Touchstone, J.A., Dosemeci, M., 2005. Potential occupational risks for neurodegenerative diseases. American Journal of Industrial Medicine 48(1), 63-77.

Pedersen, C., Poulsen, A.H., Rod, N.H., Frei, P., Hansen, J., Grell, K., Raaschou-Nielsen, O., Schüz, J., Johansen, C., 2017. Occupational exposure to extremely low-frequency magnetic fields and risk for 
central nervous system disease: an update of a Danish cohort study among utility workers. International Archives of Occupational and Environmental Health, 1-10.

Qiu, C., Fratiglioni, L., Karp, A., Winblad, B., Bellander, T., 2004. Occupational exposure to electromagnetic fields and risk of Alzheimer's disease. Epidemiology 15(6), 687-694.

Repacholi, M.H., Lerchl, A., Roosli, M., Sienkiewicz, Z., Auvinen, A., Breckenkamp, J., d'Inzeo, G., Elliott, P., Frei, P., Heinrich, S., Lagroye, I., Lahkola, A., McCormick, D.L., Thomas, S., Vecchia, P., 2012. Systematic review of wireless phone use and brain cancer and other head tumors.

Bioelectromagnetics 33(3), 187-206.

Richardson, J.R., German, D., Levey, A., 2014. Alzheimer disease risk factors. JAMA neurology 71(8), 1051-1051.

Roosli, M., 2014. Epidemiology of electromagnetic fields. Crc Press.

Roosli, M., Lortscher, M., Egger, M., Pfluger, D., Schreier, N., Lortscher, E., Locher, P., Spoerri, A., Minder, C., 2007. Mortality from neurodegenerative disease and exposure to extremely low-frequency magnetic fields: 31 years of observations on Swiss railway employees. Neuroepidemiology 28(4), 197206.

Röösli, M., Vienneau, D., 2014. Epidemiological exposure assessment, in: Röösli, M. (Ed.) Epidemiology of Electromagnetic Fields. CRC Press, pp. 35-57.

Salib, E., Hillier, V., 1996. A case-control study of Alzheimer's disease and aluminium occupation. The British journal of psychiatry : the journal of mental science 168(2), 244-249.

Savitz, D.A., Checkoway, H., Loomis, D.P., 1998a. Magnetic field exposure and neurodegenerative disease mortality among electric utility workers. Epidemiology 9(4), 398-404.

Savitz, D.A., Loomis, D.P., Tse, C.K., 1998b. Electrical occupations and neurodegenerative disease: analysis of U.S. mortality data. Archives of environmental health 53(1), 71-74.

Seidler, A., Geller, P., Nienhaus, A., Bernhardt, T., Ruppe, I., Eggert, S., Hietanen, M., Kauppinen, T., Frolich, L., 2007. Occupational exposure to low frequency magnetic fields and dementia: a case-control study. Occup Environ Med 64(2), 108-114.

Seidler, A., Geller, P., Nienhaus, A., Bernhardt, T., Ruppe, I., Eggert, S., Hietanen, M., Kauppinen, T., Frölich, L., 2007. Occupational exposure to low frequency magnetic fields and dementia: a case-control study. Occup. Environ. Med. 64(2), 108-114.

Sobel, E., Davanipour, Z., Sulkava, R., Erkinjuntti, T., Wikstrom, J., Henderson, V.W., Buckwalter, G., Bowman, J.D., Lee, P.J., 1995. Occupations with exposure to electromagnetic fields: A possible risk factor for Alzheimer's disease. American Journal of Epidemiology 142(5), 515-524.

Sobel, E., Dunn, M., Davanipour, Z., Qian, Z., Chui, H., 1996. Elevated risk of Alzheimer's disease among workers with likely electromagnetic field exposure. Neurology 47(6), 1477-1481.

Sorahan, T., Mohammed, N., 2014. Neurodegenerative disease and magnetic field exposure in UK electricity supply workers. Occupational medicine (Oxford, England) 64(6), 454-460.

Stampfer, M.J., 2009. Welding occupations and mortality from Parkinson's disease and other neurodegenerative diseases among United States men, 1985-1999. J Occup Environ Hyg 6(5), 267-272. Stampfer, M.J., 2009. Welding occupations and mortality from Parkinson's disease and other neurodegenerative diseases among United States men, 1985-1999. Journal of occupational and environmental hygiene 6(5), 267-272.

T O'Brien, J., Thomas, A., 2015. Vascular dementia. The Lancet 386(10004), 1698-1706.

Vergara, X., Kheifets, L., Greenland, S., Oksuzyan, S., Cho, Y.S., Mezei, G., 2013. Occupational exposure to extremely low-frequency magnetic fields and neurodegenerative disease: a meta-analysis. Journal of occupational and environmental medicine 55(2), 135-146.

Vina, J., Lloret, A., 2010. Why women have more Alzheimer's disease than men: gender and mitochondrial toxicity of amyloid-beta peptide. Journal of Alzheimer's disease : JAD 20 Suppl 2, S527533.

World Health Organization, 2016. Agents classified by the IARC monographs, World Health Organization, International Agency for Research on Cancer. http://monographs. iarc.

fr/ENG/Classification. Last Accessed, Last Accessed ed. 
Zhang, Y., Lai, J., Ruan, G., Chen, C., Wang, D.W., 2016. Meta-analysis of extremely low frequency electromagnetic fields and cancer risk: a pooled analysis of epidemiologic studies. Environment international 88, 36-43. 